\title{
Successful hair Regrowth in a Korean patient With alopecia universalis following tofacitinib TREATMENT
}

Singapore Med J 2017; 58(5): 279-280 doi: 10.11622/smedj.2017039

Dear Sir,

Alopecia areata (AA) is a nonscarring form of alopecia that affects approximately $1.7 \%$ of the population. ${ }^{(1)}$ In this condition, loss of hair may be localised or widespread. Severe AA is known as alopecia universalis. ${ }^{(1)}$ AA may be secondary to an autoimmune reaction involving antibodies, T-cells and cytokine-mediated losses. Recent research conducted in human subjects has identified several genes underlying AA. ${ }^{(2,3)}$ Jabbari et al identified a prominent interferon (IFN) signature in AA, which signals through Janus kinase (JAK) molecules. ${ }^{(4)}$ Tofacitinib citrate, a novel small-molecule selective JAK $1 / 3$ inhibitor for the treatment of moderate-to-severe rheumatoid arthritis, was approved by the Food and Drug Administration in late 2012. ${ }^{(5)}$ JAK inhibitors are emerging as potential therapies for many autoimmune conditions, including AA. ${ }^{(6)}$ We report a case of significant hair regrowth following treatment with oral tofacitinib citrate for alopecia universalis.

A 28-year-old woman presented to us for management of alopecia universalis. She reported a history of AA, which started at about 20 years of age and progressed to alopecia universalis within three years. Treatment with minoxidil, methotrexate and platelet-rich plasma injection had not been effective. The patient's medical history was unremarkable and she denied a family history of alopecia. On examination, she was found to have scant scalp hair with no eyebrows or facial hair, and no hair on her arms, legs, torso, axillae and groin. The results of laboratory evaluations, including antinuclear antibody, rheumatoid factor, anti-Ro antibody, anti-La antibody and anti-double stranded DNA antibody, were all negative. The patient began treatment with tofacitinib $5 \mathrm{mg}$ twice daily. Two weeks after starting treatment, the patient experienced a remarkable improvement in hair regrowth. Scalp hair had fully regrown by 32 weeks of treatment (Fig. 1). Following tofacitinib treatment, the patient had complete hair growth on her scalp, with no signs of recurrence of alopecia universalis. Laboratory monitoring showed no abnormalities in serum creatinine, electrolytes, glucose, complete blood count, hepatic function and lipid profiles. The patient did not report any side effects such as herpes zoster.

Small-molecule JAK inhibitors have been developed as novel therapeutic agents and are currently available for the treatment of myelofibrosis, rheumatoid arthritis and psoriasis. AA is known as a prominent abnormality in the IFN pathway. Type I and II IFNs signal through cell surface receptors that initially activate JAKs, specifically JAK1 and either tyrosine kinase 2 (TYK2) or JAK2, respectively. ${ }^{(4)}$ Recently, an open-label clinical trial showed that the JAK inhibitor ruxolitinib, which has relative selectivity for JAK1 and JAK2, reversed the disease in three patients with moderate-to-severe AA. ${ }^{(7)}$ Another case report showed that treatment of alopecia universalis and psoriasis using tofacitinib resulted in a clinical response. ${ }^{(5)}$ JAK-family protein kinases are downstream effectors of the IFN receptor in keratinocytes; their signalling mediates interlukin 15 activation of T-cells, which explains the effectiveness of JAK inhibitors for the treatment of AA. ${ }^{(8)}$ This class of drugs offers the convenience of being orally bioavailable when compared with biologic inhibitors of IFNs. ${ }^{(4)}$ JAK inhibitors are a promising class of drugs for treatment of AA. Although the sustainability of this treatment's effects is still unknown, ${ }^{(9)}$ this is the first report of effective tofacitinib therapy in a patient with alopecia universalis in Korea.
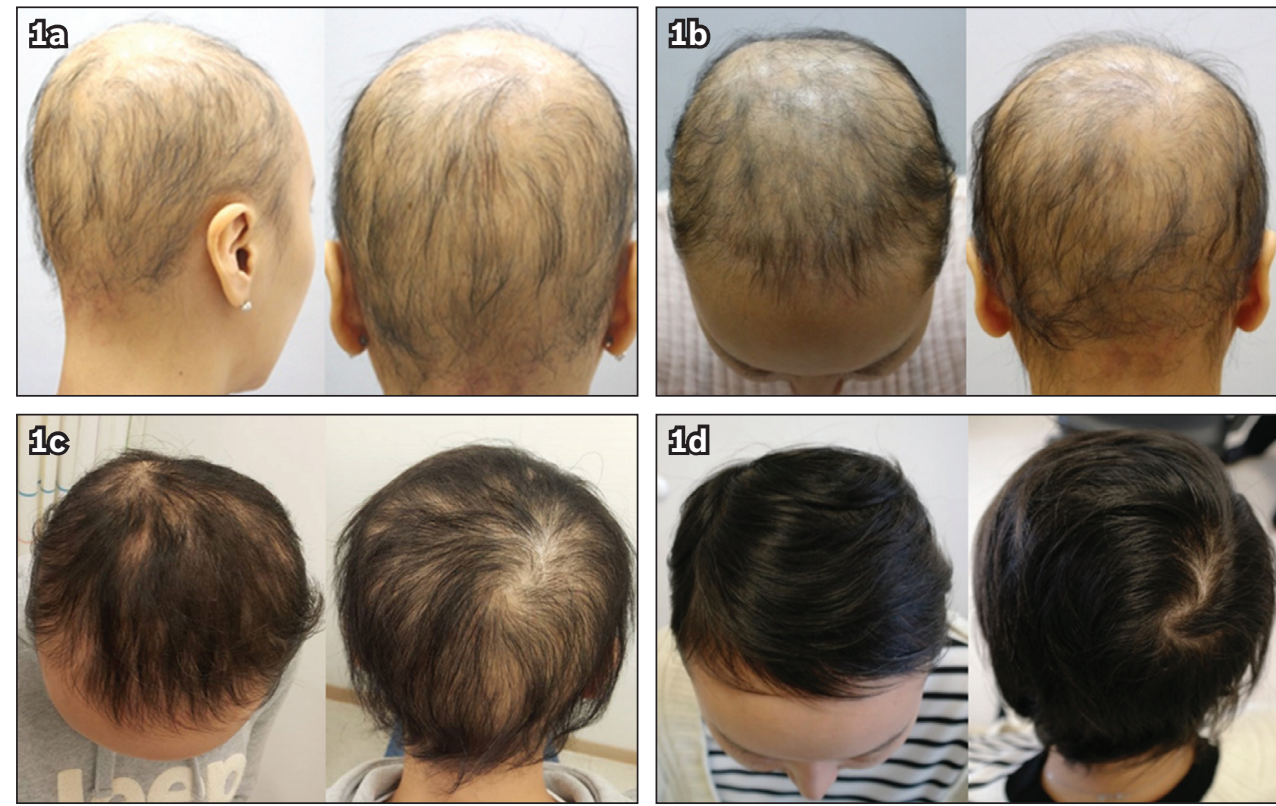

Fig. 1 Photographs show (a) the patient's scalp before treatment; (b) some improvement with partial hair growth after two weeks of tofacitinib treatment; (c) near complete regrowth of hair after six weeks of therapy; and (d) full regrowth of hair after 32 weeks of therapy. 
Yours sincerely,

Bo Young $\underline{\mathrm{Kim}}^{1}$, Hyun-Sook $\underline{\mathrm{Kim}}^{2}$

${ }^{1}$ Department of Internal Medicine, Division of Rheumatology, Gangneung Asan Hospital, Gangneung, ${ }^{2}$ Department of Internal Medicine, Division of Rheumatology, Soonchunhyang University College of Medicine, Seoul, Republic of Korea. healthyra@schmc.ac.kr

\section{References}

1. Lazzarini R, Capareli GC, Buense R, Lellis RF. Alopecia universalis during treatment with leflunomide and adalimumab - case report. An Bras Dermatol 2014; 89:320-2.

2. Betz RC, Petukhova L, Ripke S, et al. Genome-wide meta-analysis in alopecia areata resolves HLA associations and reveals two new susceptibility loci. Nat Commun 2015; 6:5966.

3. Petukhova L, Duvic M, Hordinsky M, et al. Genome-wide association study in alopecia areata implicates both innate and adaptive immunity. Nature 2010; 466:113-7.

4. Jabbari A, Dai Z, Xing L, et al. Reversal of alopecia areata following treatment with the JAK1/2 inhibitor baricitinib. EBioMedicine 2015; 2:351-5.

5. Craiglow BG, King BA. Killing two birds with one stone: oral tofacitinib reverse alopecia universalis in a patient with plaque psoriasis. J Invest Dermatol 2014; 134:2988-90.

6. Jabbari A, Nguyen N, Cerise JE, et al. Treatment of an alopecia areata patient with tofacitinib results in regrowth of hair and changes in serum and skin biomarkers. Exp Dermatol 2016; 25:642-3.

7. Xing L, Dai Z, Jabbari A, et al. Alopecia areata is driven by cytotoxic T lymphocytes and is reversed by JAK inhibition. Nat Med $2014 ; 20: 1043-9$.

8. Dhayalan A, King BA. Tofacitinib citrate for the treatment of nail dystrophy associated with alopecia universalis. JAMA Dermatol 2016; 152:492-3.

9. Anzengruber F, Maul JT, Kamarachev J, et al. Transient efficacy of tofacitinib in alopecia areata universalis. Case Rep Dermatol 2016; 8:102-6. 